Original article

\title{
Negative social comparisons and psychosis proneness in a healthy adolescent population
}

\author{
F.A. Cotier ${ }^{\mathrm{a}}$, T. Toulopoulou ${ }^{\mathrm{a}, \mathrm{b}, \mathrm{c}, *}$ \\ ${ }^{a}$ Department of Psychology, the University of Hong Kong, Pokfulam Road, Hong Kong SAR, China \\ b Department of Psychology, Bilkent University, Main Campus, Bilkent, Ankara, Turkey \\ ${ }^{\mathrm{c}}$ Department of Basic and Clinical Neuroscience, Institute of Psychiatry, Psychology and Neuroscience, King's College London, 16 De Crespigny Park, London \\ SE5 $8 A F, U K$
}

\section{A R T I C L E I N F O}

\section{Article history:}

Received 2 June 2017

Received in revised form 21 August 2017

Accepted 21 August 2017

Available online 1 September 2017

\section{Keywords:}

Social rank

Group-fit

Belonging

Psychosis

Twins

\begin{abstract}
A B S T R A C T
There is growing evidence of an association between negative social comparisons (NSC) and both psychosis, and psychosis proneness. The majority of the work thus far, however, has focused largely on one type of NSC, namely, social rank. Whilst social rank is clearly an important factor, an individual's perception of belonging is likely also of importance; particularly, when considering individuals from collectivistic cultures such as China, where greater emphasis is placed on fitting into the group. There is also limited research investigating what factors may contribute towards the relationship between NSC and psychosis proneness, and to what extent this relationship may be due to common familial factors. To address these issues, we examined whether (1) Social rank and perceived belonging predict negative, positive and depressive psychotic experiences in a Chinese, adolescent, twin and sibling population, (2) coping styles moderate the impact of these relationships and (3), there is a familial association between NSC and psychosis proneness. Both social rank and perceived belonging were found to predict the negative and depressive dimensions of psychosis. These relationships were moderated by problemfocused coping styles. Interestingly, the association between perception of belonging, and negative psychotic experiences was familial-and stronger in Monozygotic twins-indicating perhaps shared aetiology due to common genes. Our findings highlight NSC as potential vulnerability markers for negative and depressive psychotic experiences, and suggest potentially different aetiological pathways amongst different NSC and different psychotic experiences. On a clinical level, our findings emphasize the need to consider coping styles when treating at-risk individuals.
\end{abstract}

(c) 2017 Elsevier Masson SAS. All rights reserved.

\section{Introduction}

Several influential theories of mental disorder, and in particular psychosis, have highlighted the potentially important role of negative social comparisons (NSC). Gilbert and Allan [1], for example, suggested that individuals who perceive themselves as more subordinate or lower ranking compared to others are at greater risk of developing mental disorder. Similarly, in their introduction to the Social Defeat Hypothesis, Selten et al. propose perceptions of holding "a subordinate position" or "outsider status" to be significant risk factors for the development of psychosis [2]. More recently, in relation to psychosis, researchers

\footnotetext{
* Corresponding author. Department of Psychology, Bilkent University, Main Campus, Bilkent, Ankara, Turkey. Fax: +90 3122664960.

E-mail address: timothea.toulopoulou@kcl.ac.uk (T. Toulopoulou).
}

have proposed a paranoia hierarchy in which social evaluative concerns, or feelings of inadequacy compared to others, form the basis of the paranoia experience $[3,4]$.

There is now growing evidence to support these proposals implicating NSC in the development of psychosis. Negative social comparative beliefs, for example, have been found to be associated with hallucinations, such as patients' perceptions of the power of their voices [5-7]. Social rank (one form of NSC) has also been reported to predict paranoia in the general population [8] and play a key role in the transition from subclinical to clinical levels of paranoia [3,4]. Most recently, NSC have been shown to predict positive and negative psychotic experiences, and play a mediatory role between social adversity and negative psychotic experiences [9]. Using advanced modelling techniques and longitudinal approaches, several studies have also examined the directionality of these effects, and found NSC to exist before the emergence of these subclinical psychotic 
experiences [e.g. 10]. This suggests that NSC may represent a potential vulnerability marker for the development of psychosis.

Although these studies provide important preliminary evidence into the association between NSC and psychosis, the majority have tended to either use only the social "rank" scale ([e.g. 4]) or a total score comprised of the two subscales (social rank and belonging). Whilst social rank, or an individual's beliefs regarding their social standing or position in comparison to others [11], is clearly an important factor, an individual's perceptions of belonging or fitting into "the group" - another form of NSC - seems equally important to consider. This may be particularly significant when considering individuals from collectivistic cultures, such as China, who are thought to have more interdependent self-construals and thus place greater emphasis on the group and interpersonal relations [12], and consequently may be more impacted by lower perceptions of belonging. Based on these predictions, we sought to investigate how different types of NSC beliefs (rank and belonging) relate to the different dimensions of psychosis proneness (positive, negative, depressive) within a Chinese population.

In addition to further clarifying the link between NSC and psychosis proneness, it is important to determine what factors may act as moderators of this relationship, as these may represent important targets for intervention. This is particularly important given the findings of a recent study, which suggested that NSC beliefs alone may not be sufficient to induce paranoia. In an interesting study, Ascone et al. [13] induced feelings of lower rank in participants through the use of an experimental task, which mimicked social network interactions, and found social rank to affect sadness, but not paranoia. Furthermore, a significant moderation effect of fear of rejection between the experimental conditions (i.e. pre- and post-experimental manipulation of social rank) was found. These findings highlight the importance of considering which factors may exacerbate or buffer the impact of NSC on the development of psychosis.

One factor that may play a moderating role is the way in which an individual copes, or deals, with their NSC beliefs. Coping style seems to play a crucial role in the development and maintenance of psychotic symptoms in psychosis prone and at-risk individuals [14-18], and hence it is important to identify their precise role in the relationship between NSC and psychotic experiences. However, to our knowledge, no study to date has specifically examined the role of coping strategies in this relationship.

Coping styles are commonly classified into emotion-focused and problem-focused strategies [19]. Emotion-focused strategies aim at soothing the negative associated emotions and moving away from the problem (e.g. thinking reassuring thoughts). Problem-focused strategies aim instead at actively trying to solve the problem at hand and focus on the source of the problem (e.g. engaging in social activities to cope with feelings of isolation or seeking social support to feel included) [20,21]. Problem-focused strategies have been found to be associated with better mental health outcomes for adolescents [20] and more effective dealing with daily stressors [22]. Yet, individuals with early psychosis generally report more emotion-focused coping strategies [22]. We therefore sought to examine the role of coping styles in the relationship between NSC and psychosis proneness. Understanding whether different coping styles may exacerbate or buffer the harmful effects of NSC on psychosis proneness, will allow for more effective and targeted interventions.

Finally, when examining vulnerability markers for psychosis it is also important to consider possible familial influences. Specifically, whether familial (genetic and shared environmental) or non-shared environmental factors may explain this relationship, thereby opening up new avenues for translational research. Whilst little is known about the extent to which genetic and shared environmental influences (i.e. factors shared within families) may contribute to negative social comparisons, research suggests a high percentage of variance in self-esteem-a closely related conceptto be explained by such factors [23]. Furthermore, there is some evidence that other factors, which may be linked with negative social comparisons, such as stress reactivity, may congregate with psychosis proneness [24]. That is, stress reactivity and psychosis proneness seem to be explainable by common genetic influences. It therefore seems possible that negative social comparisons and psychosis proneness may also share common etiological influences including shared environmental (and potentially genetic) factors. We therefore also examined the extent to which the relationship between social comparisons and psychosis proneness is influenced by familial and non-familial factors. If familial, this could indicate that the two share, to some extent, their genetic or shared environmental aetiological factors.

Aims of the study:

- to determine how different types of social comparative beliefs (i.e. rank and belonging) are associated with the three dimensions of psychosis proneness (i.e. positive, negative and depressive) in a Chinese population;

- to examine whether coping styles moderate the impact of NSC on psychosis proneness;

- to explore whether there is a familial association between NSC and psychosis proneness.

We hypothesized that NSC will predict all three forms of psychotic experiences (negative, positive and affective). We further hypothesized that problem-focused coping styles (e.g. seeking social support) will buffer the harmful effect of NSC on psychosis proneness, and that emotion-focused strategies (e.g. thinking reassuring thoughts) will exacerbate this relationship. Finally, we hypothesized that NSC (both belonging and rank) will share familial aetiology with all three forms of psychotic experiences.

\section{Material and methods}

\subsection{Procedure}

Ethical approval was obtained from the Human Research Ethics Committee of the University of Hong Kong. Participants signed written consent before beginning the protocol, and in the case of minors, a legal guardian also provided written consent.

\subsection{Participants}

The sample included healthy, Chinese adolescents from the general population. Participants were recruited from Hong Kong, Beijing, Guangzhou, Nanjing and Qingdao. Participants from Beijing were recruited through the Chinese National twin register. Participants from Hong Kong, and all other locations in Mainland China were recruited through secondary schools and universities. Schools and universities from many regions of Hong Kong and Mainland China were included to ensure a sample truly representative of Chinese adolescents. Only non-clinical (i.e. those with no history of mental disorder or current mental disorder) participants were included. Only individuals of Chinese ethnicity who were native Chinese speakers were included.

The initial sample consisted of 201 individuals including 76 twin pairs and 49 siblings. Thirteen individuals were removed from the sample due to missing data, thus resulting in a final sample of 188 participants including 44 siblings, 70 twin pairs $(\mathrm{MZ}=37, \mathrm{DZ}=25$, Unknown $=8), 3$ twins without their co-twins 
Table 1

Descriptive statistics.

\begin{tabular}{lll}
\hline & Sample 1 $(n=188)$ & Sample 2 $(n=262)$ \\
\hline Site & & \\
$\quad$ Hong Kong ${ }^{\mathrm{a}}$ & $131(30)^{\mathrm{a}}$ & $68(21)^{\mathrm{a}}$ \\
Beijing $^{\mathrm{a}}$ & $57(70)^{\mathrm{a}}$ & $54(26)^{\mathrm{a}}$ \\
Nanjing & $\mathrm{n} / \mathrm{a}$ & $8(24)$ \\
Guangzhou & $\mathrm{n} / \mathrm{a}$ & $64(3)$ \\
Qingdao & $\mathrm{n} / \mathrm{a}$ & $68(26)$ \\
Age, mean (SD) & $17.67(1.93)$ & $17.66(1.89)$ \\
Sex, $n(\%)$ & & \\
$\quad$ Female & $124(66)$ & $167(64)$ \\
$\quad$ Male & $64(34)$ & $95(36)$ \\
CAPE negative dimension, mean (SD) & $2.07(0.59)$ & $1.90(0.55)$ \\
CAPE positive dimension & $1.32(0.52)$ & $1.25(0.40)$ \\
CAPE depressive dimension & $1.95(0.54)$ & $1.80(0.47)$ \\
SCS total & $60.10(14.2)$ & $61.53(13.31)$ \\
SCS belonging & $30.32(7.51)$ & $31.34(7.31)$ \\
SCS rank & $29.91(7.51)$ & $30.19(7.31)$ \\
\hline
\end{tabular}

SD: standard deviation; SCS: Social Comparison Scale; CAPE: Community Assessment of Psychic Experiences; n/a: not applicable.

${ }^{a}$ Indicates where there was overlap in the two samples.

(1 DZ, $2 \mathrm{MZ}$ ) and 1 lone sibling. For the question of familiarity (sample 2), only the twin pairs with complete zygosity information were included ( $n=62$ ). An additional 69 twin pairs were recruited for the purpose of this question, resulting in a total of $131(\mathrm{MZ}=83$, $\mathrm{DZ}=46$ ) twin pairs for the familiarity analyses (Table 1 ).

\subsection{Measures}

All instruments were translated, back-translated and adapted for use with Chinese participants according to existing guidelines [25].

\subsubsection{Social comparisons}

Social comparisons were assessed with the Social Comparison Scale (SCS) [11]. The SCS employs a semantic differential approach to assess an individual's perceived social rank and belongingness. The scale consists of 11 bipolar items (e.g. Inferior-Superior). Items are rated on a 10-point likert scale with lower scores indicating lower perceived belongingness and rank. The total score for the social comparison measure, which was the sum of all items, was calculated. Separate variables for belonging and social rank were also computed. The Cronbach alpha for the total scale was 0.92 and 0.87 (rank) and 0.87 (belonging) for the two subscales.

\subsubsection{Psychosis proneness}

Self-reported subclinical psychotic experiences were assessed using the Chinese version of the Community Assessment of Psychic Experiences (CAPE-C15) [26-28]. The translated version of the CAPE includes three subscales (Positive, Negative, Depressive) consisting of 4, 5 and 6 items respectively. An example item is: "Do

你有否曾經覺得好像有一個針對你的陰謀? /你有否曾经觉得好像有一个针对你的阴谋? ’’

you ever feel there is a conspiracy against you?/

Further description of the CAPE-C15 and the translation process can be found in our labs previous report [26]. In the current sample the CAPE-C15 reached an internal consistency of Cronbach Alpha 0.86 , representing good/excellent reliability, and the positive, negative and depressive subscales had a Cronbach alpha coefficient of $0.79,0.71$, and 0.79 , respectively. Frequency scores were rated on a 4-point Likert scale ranging from never to nearly always. Mean frequency scores were calculated for each of the three dimensions with higher scores indicating greater proneness to that dimension.
In order to normalize the distribution of the mean frequency scores, log transformations were performed.

\subsubsection{Coping styles}

The Utrecht Coping list (UCL) (Schreurs, Van de Willige, Tellegen, \& Brosschot, 1993) was adopted here to measure coping styles. This 44-item instrument measures seven scales including: active tackling, seeking social support, palliative reacting, avoiding, passive reacting, reassuring thoughts and expression of emotions (see Table 3). Of these the first two are considered as problemfocused coping strategies (i.e. actively trying to solve the problem) and the latter 5 are considered to be emotion-focused (i.e. soothing negative associated emotions through moving away from the problem). Participants were asked to indicate how they would typically respond to a problem. For example, "You see problems as a challenge/你把难题当作挑战/你把難題當作挑戰” (Problem-focused) and "You avoid difficult situations as much as possible/你会尽量避免困境/你會盡量避免困境” (Emotionfocused). The internal consistency of this questionnaire for the current sample was good to excellent with a Cronbach Alpha coefficient of 0.90 for the total scale. The reliability coefficients for the individual scales were 0.84 (active tackling), 0.69 (palliative reacting), 0.64 (avoiding), 0.81 (seeking social support) 0.76 (depressive reactive pattern), 0.61 (expression of emotions) and 0.62 (reassuring thoughts), which were in line with previously established internal consistency estimates for western populations (Schreurs et al., 1993).

\subsection{Statistical analyses}

All analyses were conducted in SPSS 20 for Mac. Given the hierarchical structure of the data, multilevel regression analysis was performed with individuals (level 1) nested within-twin pairs (level 2) for all cross-trait, within-twin analyses. All hierarchical models were conducted using the MIXED command.

\subsubsection{Associations between negative social comparisons and} psychotic experiences and coping styles as moderators (cross-trait, within-twin analyses)

2.4.1.1. Main effects. In order to examine the main effects of perceived social comparison on psychosis proneness within individuals, separate multilevel models were conducted with the three measures of psychosis proneness as the dependent variables (DV's: positive, negative and depressive) and the three measures of social comparison as independent variables (IV: total SCS, belongingness, rank).

2.4.1.2. Moderation analyses. In order to investigate the moderating role of coping strategies on the relationship between social comparisons and psychotic experiences, the seven coping style measures were individually added to the Total Social Comparison within person models as moderators. The primary focus was therefore the interaction term. Moderators were only added to models that were found to be significant in the first round of analyses.

\subsubsection{Familial associations between negative social comparisons and} psychotic experiences (cross-trait, cross-twin associations)

2.4.2.1. Main effects. Cross-trait cross-twin associations were examined through the use of simple linear regression models. Specifically, we assessed whether the three social comparison measures in twin 1 would predict the three dimension of psychosis proneness in twin 2. The IV's were the three social comparison variables in twin 1 and the DV's were the three dimensions of 
Table 2

Associations between negative social comparisons and psychotic experiences.

\begin{tabular}{|c|c|c|c|c|c|c|c|c|c|}
\hline & \multicolumn{3}{|c|}{$\begin{array}{l}\text { CAPE positive } \\
\text { Dimension }\end{array}$} & \multicolumn{3}{|c|}{$\begin{array}{l}\text { CAPE negative } \\
\text { Dimension }\end{array}$} & \multicolumn{3}{|c|}{$\begin{array}{l}\text { CAPE depressive } \\
\text { Dimension }\end{array}$} \\
\hline & B & $95 \% \mathrm{CI}$ & $P$ & B & $95 \% \mathrm{CI}$ & $P$ & B & $95 \% \mathrm{CI}$ & $P$ \\
\hline Rank & -0.004 & -0.010 to 0.002 & 0.180 & -0.014 & -0.019 to -0.009 & 0.000 & -0.012 & -0.018 to -0.008 & 0.000 \\
\hline Belonging & -0.005 & -0.01 to 0.0006 & 0.080 & -0.015 & -0.02 to -0.01 & 0.000 & -0.012 & -0.017 to -0.007 & 0.000 \\
\hline Total SCS & -0.002 & -0.01 to 0.18 & 0.125 & -0.008 & -0.01 to -0.005 & 0.000 & -0.007 & -0.009 to -0.004 & 0.000 \\
\hline
\end{tabular}

SCS: Social Comparison Scale; CAPE: Community Assessment of Psychic Experiences. The bold text indicates that the $P$ value is $<0.05$, and thus significant

psychosis in twin 2. Twins were randomly assigned an ID of either "twin 1" or "twin 2". Models were adjusted for the corresponding psychosis dimension in twin 1 [24].

2.4.2.2. Moderation analyses. In order to examine whether genetic factors may explain any familial associations found, we examined the two-way interaction between the different forms of negative social comparisons in twin 1 (those which were found to be significant predictors) $\times$ Zygosity (with two levels; MZ \& DZ). In the case that a stronger familial association was found between twin 1 negative social comparisons and twin 2 dimensions of psychosis in MZ twins it can be assumed that a genetic correlation exists. Zygosity was only examined as a moderator in those relationships that were found to be familial in nature.

\section{Results}

3.1. Associations between negative social comparisons and psychotic experiences and coping styles as moderators

\subsubsection{Main effects}

The cross-trait within-twin analyses revealed that all measures of social comparison significantly predicted the negative and depressive dimensions of psychosis proneness (Table 2). Whilst there was a non-statistical trend for the association between belongingness and the positive dimension, neither of the measures of social comparison were found to significantly predict the positive psychosis dimension (Table 2). These results therefore suggest that NSC are associated with the negative and depressive dimensions of psychosis proneness.

\subsubsection{Moderation analyses}

The moderation analysis revealed active coping to be a significant moderator of the relationship between perceived social comparisons, and both the negative and depressive dimensions of psychosis proneness. Social support was found to be a moderator for the association between NSC and the depressive dimension.
More specifically, both social support and active coping were found to buffer the harmful effects of NSC on psychosis proneness. None of the emotion-focused coping strategies were found to act as significant moderators (see Table 3 for detailed results).

\subsection{Familial associations between negative social comparisons and psychotic experiences}

\subsubsection{Main effects}

The cross-trait, cross-twin analyses revealed all three measures of perceived social comparisons in twin 1 to significantly predict negative psychotic experiences in the co-twin. Sense of belonging in twin 1 also significantly predicted depressive psychotic experiences in the co-twin. Sense of belonging and total social comparisons in twin 1 remained statistically significant predictors of negative psychotic experiences in twin 2 after adjusting for negative psychotic experiences in twin 1. Sense of belonging in twin 1 did not, however, remain a statistically significant predictor of depressive psychotic experiences in twin 2 after controlling for depressive psychotic experiences in twin 1 (see Table 4).

\subsubsection{Moderation analyses}

Zygosity was found to be a significant moderator of the relationship between total social comparisons in twin 1 and negative psychotic experiences in twin 2 with a statistically significant increase in total variation explained of $6.7 \%, F(1$, $126)=9.659, P=0.002$. Specifically, the association was stronger in MZ twins $(B=-0.10 ; P<0.01,95 \% \mathrm{CI}[-0.015,-0.005])$ compared to DZ twins $(B=0.001 ; P=0.763,95 \% \mathrm{CI}[-0.004,0.006])$. Similarly, zygosity also moderated the relationship between sense of belonging in twin 1 and negative psychotic experiences in twin 2 as evidenced by a statistically significant increase in total variation explained of $5.4 \%, F(1,125)=7.984, P=0.005$ with a greater association found in MZ twins $(B=-0.020 ; P<0.01,95 \% \mathrm{CI}$ $[-0.028,-0.011])$ compared to DZ twins $(B=-0.001 ; P=0.809$, 95\% CI [-0.011, 0.009]).

Table 3

Results of moderation analysis.

\begin{tabular}{|c|c|c|c|c|c|c|c|c|c|}
\hline & \multirow[t]{2}{*}{ Mean } & \multirow[t]{2}{*}{ SD } & \multirow[t]{2}{*}{ Min-Max } & \multicolumn{3}{|c|}{$\begin{array}{l}\text { CAPE negative } \\
\text { Dimension }\end{array}$} & \multicolumn{3}{|c|}{$\begin{array}{l}\text { CAPE depressive } \\
\text { Dimension }\end{array}$} \\
\hline & & & & B & $95 \% \mathrm{CI}$ & $P$ & B & $95 \% \mathrm{CI}$ & $P$ \\
\hline \multicolumn{10}{|l|}{ Problem-focused } \\
\hline Active tackling & 18.37 & 3.79 & $8-28$ & 0.0008 & 0.00009 to 0.002 & 0.027 & 0.0008 & 0.0001 to 0.001 & 0.017 \\
\hline Seeking social support & 15.12 & 3.54 & $7-24$ & 0.0007 & -4.5233 to 0.0015 & 0.065 & 0.001 & 0.0003 to 0.002 & 0.003 \\
\hline \multicolumn{10}{|l|}{ Emotion-focused } \\
\hline Palliative reacting & 19.63 & 3.88 & $6-30$ & 0.0002 & -0.0004 to 0.0009 & 0.524 & 0.0005 & -0.0001 to 0.0011 & 0.116 \\
\hline Avoiding & 19.50 & 3.25 & $11-30$ & 0.0004 & -0.0002 to 0.0012 & 0.191 & 0.0004 & -0.0002 to 0.0011 & 0.198 \\
\hline Passive reacting & 12.45 & 3.56 & $0-23$ & 0.0004 & -0.0003 to 0.0011 & 0.213 & 0.0001 & -0.0005 to 0.0007 & 0.709 \\
\hline Reassuring thoughts & 12.69 & 2.61 & $6-20$ & 0.0003 & -0.0006 & 0.558 & 0.0003 & -0.0005 to 0.0011 & 0.462 \\
\hline Expression of emotions & 6.47 & 1.84 & $3-11$ & 0.0010 & -0.0004 to 0.0025 & 0.160 & 0.0002 & -0.0011 to 0.0015 & 0.821 \\
\hline
\end{tabular}

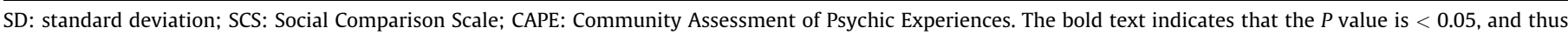
significant. 
Table 4

Familial associations between negative social comparisons and psychotic experiences.

\begin{tabular}{|c|c|c|c|c|c|c|}
\hline & \multicolumn{3}{|l|}{ Twin 1} & \multicolumn{3}{|l|}{ Twin 2} \\
\hline & \multicolumn{3}{|c|}{$\begin{array}{l}\text { CAPE: negative psychosis } \\
\text { dimension }\end{array}$} & \multicolumn{3}{|c|}{$\begin{array}{l}\text { CAPE: depressive psychosis } \\
\text { dimension }\end{array}$} \\
\hline & $B$ & $\mathrm{t}$ & $P$ & $B$ & $\mathrm{t}$ & $P$ \\
\hline \multicolumn{7}{|l|}{ Unadjusted } \\
\hline Rank & -0.007 & -2.06 & 0.041 & -0.004 & -1.24 & 0.218 \\
\hline Belonging & -0.012 & -3.60 & 0.000 & -0.006 & -2.12 & 0.036 \\
\hline Total SCS & -0.005 & -2.83 & 0.005 & -0.003 & -1.67 & 0.098 \\
\hline \multicolumn{7}{|l|}{ Adjusted } \\
\hline Rank & -0.006 & -1.57 & 0.120 & -0.002 & -0.609 & 0.544 \\
\hline Belonging & -0.011 & -3.12 & 0.002 & -0.004 & -1.19 & 0.234 \\
\hline Total SCS & -0.005 & -2.32 & 0.022 & -0.001 & -0.858 & 0.392 \\
\hline
\end{tabular}

SCS: Social Comparison Scale; CAPE: Community Assessment of Psychic Experiences. The bold text indicates that the $P$ value is $<0.05$, and thus significant.

\section{Discussion}

This study examined the relationship between perceived social comparisons and psychosis proneness in ethnically Chinese individuals. To our knowledge this was the first study to explore associations between different types of social comparisons (i.e. rank and belongingness) and dimensions of psychosis proneness. In addition it was the first study to examine coping styles as potential moderator factors, and familial associations between NSC and psychosis proneness. Three major findings were revealed: firstly, both belongingness and rank were found to predict negative and depressive psychotic experiences, but not positive psychotic experiences. Secondly, problem-focused coping styles were found to buffer the impact of negative social comparisons on psychotic experiences. Finally, a familial association was found between belonging and negative psychotic experiences, and this relationship was stronger in monozygotic twins, suggesting perhaps shared aetiology due to common genes. These findings shed light on the exact nature of the relationship between NSC and psychosis proneness, and have implications for early intervention and future translational studies in the field of psychosis.

As was hypothesized, negative social comparisons, including both belongingness and rank, significantly predicted the negative and depressive dimensions of psychosis proneness. These findings are consistent with a study, which found experimentally induced social rank to lead to significant increases in sadness [13]. These findings are also in line with studies, which have found associations between negative interpersonal concepts and negative symptoms in psychosis [29], as well as the research suggesting a central role for interpersonal insecurities in the development of depressive and anxious symptoms $[1,30]$. If an individual feels left out or views himself/herself more negatively in comparison to others, it makes sense that the individual may be less motivated or experience less pleasure in engaging in such activities [29].

Our hypothesis regarding the positive dimension of psychosis proneness was not supported. Even though this finding is consistent with at least one other study [13], several other studies have found associations between NSC and paranoia at a subclinical level $[3,4,8]$ as well as between NSC and hallucinations [5-7]. There are several potential explanations for these findings. One possibility is that NSC are simply an indirect measure of social adversity and that, in terms of positive symptoms, the actual relationship is with social adversity not NSC [9]. This would also explain why previous work ([e.g. 8]) which did not consider social adversity found a significant relationship, and why Jaya et al. [9] failed to find a relationship between social rank and positive symptoms when they considered it as a mediator between social adversity and positive symptoms. Another potential explanation is that these findings reflect an affective pathway as postulated by key cognitive models of psychosis ([e.g. 31]). That is, NSC may lead to increased negative emotion e.g. sadness, which in turn leads to positive symptoms [13].

Consistent with our hypotheses, more frequent use of problemfocused coping styles including active coping and social support seeking were found to buffer the harmful effect of NSC on psychotic experiences. These findings are consistent with prior research that highlights the protective role of more proactive problem-focused coping styles against the development of psychopathology [20]. These findings also support cognitive models that propose a protective role for problem-focused coping strategies in the genesis of psychotic disorder [32]. Emotion-focused strategies did not seem to exacerbate the relationship between NSC and psychotic experiences. Prior research suggested that more maladaptive coping strategies may moderate (specifically, exacerbate) the relationship between stressful experiences and the development of psychosis, as in the study by Phillips et al. [16], which found the coping strategies employed rather than the stressful experiences per se to predict psychosis. Our findings on the other hand suggest that emotion-focused strategies neither exacerbate nor buffer the relationship between NSC and psychosis proneness. One possibility is that emotion-focused strategies may only play a role once the psychotic symptoms reach a certain level of severity. This would explain why the study by Philips et al. [16], for example, found maladaptive coping styles to play a moderately role. Another possible explanation is that, as the literature referred to thus far has focused on western populations, this finding may reflect cultural differences. That is, using emotion-focused strategies (i.e. those that move away from the problem with the aim of soothing negative associated emotions) may be less detrimental, in terms of psychosis, in Chinese populations. Future research may wish to explore cultural differences in how coping styles impact the development of psychosis proneness.

Belonging and total social comparison in the first twin were associated with negative psychotic experiences in the second twin, thereby suggesting a familial association between an individual's sense of belonging and negative psychotic experiences. Furthermore, this relationship was found to be stronger amongst MZ twins suggesting it may be due to shared genetic influences. Yet, no familial association was found between depressive psychotic experiences in twin one and NSC in twin two. These findings suggest possible etiological differences in the route to the negative and depressive dimensions of psychosis. More specifically, belonging and negative psychotic experiences seem to share genetic and environmental influences that are common to members of the same family, whilst NSC and depressive symptoms appear to share unique or idiosyncratic environmental factors. Researchers have highlighted the possibility of different routes to different forms of psychosis. Myin-Germeys and van Os [31], for example, suggested one pathway to the positive syndrome, nondeficit, good outcome form of schizophrenia I, characterized primarily by altered stress sensitivity, and another to the negative syndrome, deficit poor outcome Schizophrenia II, characterized primarily by cognitive impairment [31]. These differences in familial associations thus likely reflect such differences, perhaps even suggesting a third pathway to the more depressive form of schizophrenia: Schizoaffective disorder. Alternatively, depressive symptoms may develop as the result of distress associated with the negative and positive symptoms [27].

The findings of the current study should be interpreted in light of its limitations. Firstly, the cross-sectional nature of the study does not allow for the direction of causality to be established. It is possible that individuals with increased psychosis proneness, particularly subclinical paranoia, may lead to more NSC. On the other hand, NSC can be a vulnerability marker for the later 
development of psychosis. Future studies may wish to employ a longitudinal design to confirm the direction of causality. A second limitation is that measures in this study involved subjective reports and thus may be influenced by self-report biases. Our questionnaires nonetheless have been found to have high reliability and validity, particularly for adolescent samples such as the one examined here $[11,33,34]$, so the present findings are unlikely to be primarily due to self-report biases. Thirdly, we were not able to employ twin modelling due to the limited sample size. The results do, however, justify the implementation of this approach in a larger sample in order to determine whether the established familial associations can be explained by shared environmental or genetic factors and quantify the extent. Finally, whilst some of the present findings were interpreted in terms of cross-cultural differences in self-construals, no such measure had been taken. On the other hand, there is substantial evidence for more interdependent self-construals in individuals in collectivist societies such as China and Hong Kong than in the more individualistic Western societies [12]. Future research may consider direct comparisons of individuals from Chinese and Western cultures, including measures of self-construals, to further evaluate these hypotheses.

Despite these limitations, the present research has important implications for both early intervention and further translational research. Firstly, these findings speak directly to the importance of using cognitive behaviour therapy approaches which place strong emphasis on the development of more effective coping strategies and more positive interpersonal schemas with at-risk individuals. Our findings suggest that guiding at-risk individuals to engage in problem-focused coping strategies may be particularly important as they may help buffer the potentially negative effects of NSC and, as a result, hinder the development of full-blown psychotic symptoms. Secondly, these findings tentatively suggest that measures of NSC may inform assessments for the early identification of at-risk individuals in Chinese populations. This, however, would need to be confirmed in future studies. Thirdly, our findings suggest a need for further work confirming the relationship between negative social comparisons and psychosis proneness/ Psychosis and it's precise nature using genetic modelling and longitudinal approaches. Finally, this work highlights the need to consider cross-cultural differences (particularly individualistic vs. collectivistic cultures) in pathways to psychosis and encourages further research in this regard.

\section{Disclosure of interest}

The authors declare that they have no competing interest.

\section{References}

[1] Gilbert P, Allan S. The role of defeat and entrapment (arrested flight) in depression: an exploration of an evolutionary view. Psychol Med 1998;28:585-98.

[2] Selten JP, Cantor-Graae E. Social defeat: risk factor for schizophrenia? Br J Psychiatry 2005;187:101-2.

[3] Bebbington PE, McBride O, Steel C, Kuipers E, Radavanovic M, Brugha T, et al. The structure of paranoia in the general population. $\mathrm{Br} \mathrm{J}$ Psychiatry 2013;202:419-27.

[4] Freeman D, Garety PA, Bebbington PE, Smith B, Rollinson R, Fowler D, et al. Psychological investigation of the structure of paranoia in a non-clinical population. Br J Psychiatry 2005;186:427-35.

[5] Birchwood M, Gilbert P, Gilbert J, Trower P, Meaden A, Hay J, et al. Interpersonal and role-related schema influence the relationship with the dominant 'voice' in schizophrenia: a comparison of three models. Psychol Med 2004;34:1571-80.
[6] Birchwood M, Meaden A, Trower P, Gilbert P, Plaistow J. The power and omnipotence of voices: subordination and entrapment by voices and significant others. Psychol Med 2000;30:337-44.

[7] Gilbert P, Birchwood M, Gilbert J, Trower P, Hay J, Murray B, et al. An exploration of evolved mental mechanisms for dominant and subordinate behaviour in relation to auditory hallucinations in schizophrenia and critical thoughts in depression. Psychol Med 2001;31:1117-27.

[8] Freeman D, Nicole E, Lister R, Antley A, Dunn G, Slater M. Height, social comparison, and paranoia: an immersive virtual reality experimental study. Psychiatry Res 2014;218:348-52.

[9] Jaya ES, Ascone L, Lincoln TM. Social adversity and psychosis: the mediating role of cognitive vulnerability. Schizophr Bull 2016;43:557-65.

[10] Fowler D, Hodgekins J, Garety P, Freeman D, Kuipers E, Dunn G, et al. Negative cognition, depressed mood, and paranoia: a longitudinal pathway analysis using structural equation modeling. Schizophr Bull 2012;38:1063-73.

[11] Allan S, Gilbert P. A Social Comparison Scale: psychometric properties and relationship to psychopathology. Pers Individual Differences 1995;19:293-9.

[12] Pfundmair M, Aydin N, Du H, Yeung S, Frey D, Graupmann V. Exclude me if you can: cultural effects on the outcomes of social exclusion. J Cross Cult Psychol 2014;46:579-96.

[13] Ascone L, Jaya ES, Lincoln TM. The effect of unfavourable and favourable social comparisons on paranoid ideation: an experimental study. J Behav Ther Exp Psychiatry 2016;56:97-105.

[14] Jalbrzikowski M, Sugar CA, Zinberg J, Bachman P, Cannon TD, Bearden CE. Coping styles of individuals at clinical high risk for developing psychosis. Early Interv Psychiatry 2012;8:68-76.

[15] Melo SS, Bentall RP. Coping in subclinical paranoia: a two nations study. Psychol Psychother 2010;83:407-20.

[16] Phillips LJ, Edwards J, McMurray N, Francey S. Comparison of experiences of stress and coping between young people at risk of psychosis and a non-clinical cohort. Behav Cogn Psychother 2012;40:69-88.

[17] Schuldberg D, Karwacki SB, Burns GL. Stress, coping, and social support in hypothetically psychosis prone subjects. Psychol Rep 1996;78:1267-83.

[18] Zong JG, Chan RC, Stone WS, Hsi X, Cao XY, Zhao Q, et al. Coping flexibility in young adults: comparison between subjects with and without schizotypal personality features. Schizophr Res 2010;122:185-92.

[19] Folkman S, Lazarus RS. If it changes it must be a process: study of emotion and coping during three stages of a college examination. J Pers Soc Psychol 1985;48:150-70.

[20] Compas BE, Connor-Smith JK, Saltzman H, Thomsen AH, Wadsworth ME Coping with stress during childhood and adolescence: problems, progress, and potential in theory and research. Psychol Bull 2001;127:87-127.

[21] Muris P, Mayer B, Reinders E, Wesenhagen C. Person-related protective and vulnerability factors of psychopathology symptoms in non-clinical adolescents. Community Ment Health J 2009;47:47-60.

[22] Macdonald EM, Elspeth M, Pica S, Mcdonald S, Hayes RL, Baglioni Jr AJ. Stress and coping in early psychosis: role of symptoms, self-efficacy, and social support in coping with stress. Br J Psychiatry 1998;172:122-7.

[23] Raevuori A, Dick D, Keski-Rahkonen A, Pulkkinen L, Rose RJ, Rissanen A, et al. Genetic and environmental factors affecting self-esteem from age 14 to 17 : a longitudinal study of Finnish twins. Psychol Med 2007:37:1625-33.

[24] Lataster T, Wichers M, Jacobs N, Mengelers R, Derom C, Thiery E, et al. Does reactivity to stress cosegregate with subclinical psychosis? A general population twin study. Acta Psychiatr Scand 2008;119:45-53.

[25] Sperber AD. Translation and validation of study instruments for cross-cultural research. Gastroenterology 2004;126:S124-8.

[26] Mark W, Toulopoulou T. Validation of the Chinese version of Community Assessment of Psychic Experiences (CAPE) in an adolescent population. Asian J Psychiatry 2017;26:58-65.

[27] Stefanis NC, Hanssen M, Smirins NK, et al. Evidence that three dimensions of psychosis have a distribution in the general population. Psychol Med 2002;32:347-58.

[28] Mark W, Toulopoulou T. Psychometric properties of "community assessment of psychic experiences": review and meta-analyses. Schizophr Bull 2016:42:34-44.

[29] Lincoln TM, Mehl S, Kesting ML, Rief W. Negative symptoms and social cognition: identifying targets for psychological interventions. Schizophr Bull 2011;37:S23-32.

[30] Gilbert P, Allan S, Brough S, Melley S, Miles JNV. Relationship of anhedonia and anxiety to social rank, defeat and entrapment. J Affect Disord 2002;71:141-51.

[31] Myin-Germeys I, van Os J. Stress reactivity in psychosis: evidence for an affective pathway to psychosis. Clin Psychol Rev 2007:27:409-24.

[32] Nuechterlein KH, Dawson ME, Ventura J, Gitlin M, Subotnik KL, Snyder KS, et al. The vulnerability/stress model of schizophrenic relapse: a longitudinal study. Acta Psychiatr Scand 1994;89:58-64.

[33] Schreurs PJG, Van de Willige G, Tellegen B, Brosschot JF. Herziene handleiding Utrechtse Coping Lijst (UCL). Lisse: Swets \& Zeitlinger, B.V; 1993.

[34] Turner H, Bryant-Waugh R, Peveler R, Bucks RS. A psychometric evaluation of an English version of the Utrecht Coping List. Eur Eating Disord Rev 2012;20:339-42. 